\title{
Decoupled and Energy Stable Time-Marching Scheme for the Interfacial Flow with Soluble Surfactants
}

\author{
Guangpu Zhu (D) and Aifen $\mathrm{Li}^{(\bowtie)}$ \\ School of Petroleum Engineering, China University of Petroleum (East China), \\ Qingdao 266580, China \\ Aifenli64@gmail.com
}

\begin{abstract}
In this work, we develop an efficient energy stable scheme for the hydrodynamics coupled phase-field surfactant model with variable densities. The thermodynamically consistent model consists of two Cahn-Hilliard-type equations and incompressible Navier-Stokes equation. We use two scalar auxiliary variables to transform nonlinear parts in the free energy functional into quadratic forms, and then they can be treated efficiently and semi-implicitly. A splitting method based on pressure stabilization is used to solve the NavierStokes equation. By some subtle explicit-implicit treatments to nonlinear convection and stress terms, we construct a first-order energy stable scheme for the two-phase system with soluble surfactants. The developed scheme is efficient and easy-to-implement. At each time step, computations of phase-field variables, the velocity and pressure are decoupled. We rigorously prove that the proposed scheme is unconditionally energy stable. Numerical results confirm that our scheme is accurate and energy stable.
\end{abstract}

Keywords: Surfactant $\cdot$ Interfacial flow $\cdot$ Phase-field modeling $\cdot$ Navier-stokes

\section{Introduction}

Surfactants, interface active agents, are known to lower the interfacial tension and allow for the formation of emulsion [1, 2]. Commonly-used surfactants are amphiphilic compounds, meaning they contain both hydrophilic heads and hydrophobic tails [1, 3]. This special molecular composition enables surfactants to selectively absorb on fluid interfaces. Surfactants play a crucial role in everyday life and many industrial processes, such as the cleanser essence, the crude oil recovery and pharmaceutical materials, thus having an understanding of their behavior is a necessity. Numerical simulation is taking an increasingly significant position in investigating the interfacial phenomena, as it can provide easier access to some quantities such as surfactant concentration, pressure and velocity, which are difficult to measure experimentally. However, the computational modeling of interfacial dynamics with surfactants remains a challenging task. 
The phase-field model is an effective modeling and simulation tool in investigating interfacial phenomena and it has been extensively used with much successes [4]. This method introduces a phase-field variable to distinguish two pure phases. The interface is treated as a thin layer, inside which the phase-field variable varies continuously [5, 6]. Unlike shape interface models, the phase-field model does not need to track the interface explicitly, and the interface can be implicitly and automatically captured by the evolution of phase-field variable. Therefore, the computations and analysis of the phase-field model are easier than other models [7, 8].

The phase-field model was first used to study the dynamics of phase separation with surfactants in [9]. Two phase-field variables were introduced in their work. Since then, a variety of phase-field surfactant models have been proposed and reviews of these models can refer to [10-12]. Here we only highlight two representative works. The authors in [13] introduced the logarithmic Floy-Huggins potential to restrict the range of surfactant concentration. A nonlinear coupling surface energy potential was used to account for the high surfactant concentration along the fluid interface. An enthalpic term was also adopted to stabilize the phase-field model and control the surfactant solubility in the bulk phases. Their model can describe realistic adsorption isotherms, e.g., Langmuir isotherm, in thermodynamic equilibrium. In [14], the authors analyzed the well-posedness of the phase-field surfactant model proposed in [13], and provided strong evidence that the model was mathematically ill-posed for a large set of physically relevant parameters. They made critical modifications to the model and substantially increased the domain of validity. In this study, we will use this modified model to describe a binary fluid-surfactant system.

Numerically, it is a challenging issue to discretize the strong couplings between two phase-field variables. The introduction of hydrodynamics will further increase the complexity for the development of numerical schemes. Several attempts have been made to solve the interfacial flows with surfactants [15-18], but none of them can provide the energy stability for numerical schemes in theory. Most recently, we constructed a first-order and a second-order schemes, which are linear and totally decoupled, for a phase-field surfactant model with fluid flow [19]. However, this study only considered the case of matched density and viscosity, which greatly reduces difficulties in algorithm developments. Thus, the main purpose of this study is to construct an efficient, easy-to-implement and energy stable scheme for the hydrodynamics coupled phase-field surfactant model with variable densities.

The rest of this paper is organized as follows. In Sect. 2, we describe a hydrodynamics coupled phase-field surfactant model with variable densities. In Sect. 3, we develop an efficient energy stable scheme carry out the energy stability for the proposed scheme. Several numerical experiments are investigated in Sect. 4 and the paper is finally concluded in Sect. 5 .

\section{Governing Equation}

In this section, we consider a typical phase-field surfactant model in [14, 19] for a twophase system with surfactants 


$$
E_{f}(\mathbf{u}, \phi, \psi)=\int\left(\frac{\mathrm{Cn}^{2}}{4}|\nabla \phi|^{2}+F(\phi)+\operatorname{Pi} G(\psi)-\frac{\psi\left(\phi^{2}-1\right)^{2}}{4}+\frac{\psi \phi^{2}}{4 \mathrm{Ex}}\right) \mathrm{d} \Omega,
$$

where $F(\phi)$ is the double well potential and $G(\psi)$ the logarithmic Flory-Huggins potential,

$$
F(\phi)=\frac{\left(\phi^{2}-1\right)^{2}}{4}, \quad G(\psi)=\psi \ln \psi+(1-\psi) \ln (1-\psi) .
$$

Two phase-field variables are used in the free energy functional. The first phase-field variable $\phi$ uses two constants ( -1 and 1) to distinguish two phases, and it varies continuously across the interface between -1 and 1 . The other phase-field variable $\psi$ is used to represent the surfactant concentration. The parameter $\mathrm{Cn}$ determines the interfacial thickness and $\mathrm{Pi}$ is a temperature-dependent parameter. More details of the free energy functional can refer to [6] and [19].

Although both the double well potential and the Flory-Huggins potential are bounded from below, the latter is not always positive in the whole domain. Thus, we add a zero term $\mathrm{Pi} B-\mathrm{Pi} B$ to the free energy functional, and rewrite (2.1) into

$$
\begin{aligned}
E_{f}(\phi, \psi)= & \int\left(\frac{\mathrm{Cn}^{2}}{4}|\nabla \phi|^{2}+F(\phi)+\operatorname{Pi}(G(\psi)+B)+\frac{\psi \phi^{2}}{4 \mathrm{Ex}}-\frac{\psi\left(\phi^{2}-1\right)^{2}}{4}\right) d \Omega \\
& -\operatorname{Pi} B|\Omega|,
\end{aligned}
$$

where the positive constant $B$ ensures $G(\psi)+B>0$, and $B=1$ is adopted in this study. Note that the free energy is not changed due to the introduction of the zero term $\mathrm{Pi} B-\mathrm{Pi} B$. We now use the scalar auxiliary variable (SAV) approach $[12,20]$ to transform the free functional into a new form. Through the simple substitution of scalar variables, the nonlinear parts of the free energy are transformed into quadratic forms of new scalar variables. More precisely, we define two scalar variables

$$
U=\sqrt{E_{u}(\phi)}, \quad V=\sqrt{E_{v}(\psi)},
$$

Where

$$
E_{u}(\phi)=\int F(\phi) d \Omega, \quad E_{v}(\psi)=\int(G(\psi)+B) d \Omega
$$

Then the free energy can be transformed into

$$
E_{f}(\phi, \psi, U, V)=\int\left(\frac{\mathrm{W} \mathrm{e}}{2} \rho|\mathbf{u}|^{2}+\frac{\mathrm{Cn}^{2}}{4}|\nabla \phi|^{2}-\frac{\psi\left(\phi^{2}-1\right)^{2}}{4}+\frac{\psi \phi^{2}}{4 \mathrm{Ex}}\right) d \Omega+U^{2}+\operatorname{Pi} V^{2}-\operatorname{Pi} B|\Omega|,
$$


Through the functional derivatives of $E_{f}$ with respect to phase-field variables $\phi$ and $\psi$, we can obtain chemical potentials $w_{\phi}$ and $w_{\psi}$

$$
\begin{gathered}
w_{\phi}=-\frac{\mathrm{Cn}^{2}}{2} \Delta \phi+\frac{U}{\sqrt{E_{u}(\phi)}} F^{\prime}(\phi)+\frac{\psi \phi}{2 \mathrm{Ex}}-\psi \phi W, U_{t}=\frac{1}{2 \sqrt{E_{u}(\phi)}} \int F^{\prime}(\phi) \phi_{t} d \Omega \\
w_{\psi}=\frac{\operatorname{Pi} V}{\sqrt{E_{v}(\psi)}} G^{\prime}(\psi)+\frac{\phi^{2}}{4 \mathrm{Ex}}-\frac{W^{2}}{4}, \quad V_{t}=\frac{1}{2 \sqrt{E_{v}(\psi)}} \int G^{\prime}(\psi) \psi_{t} d \Omega
\end{gathered}
$$

Note that $\phi^{2}-1$ are denoted as $W$ in (2.5) and (2.6).

Evolutions of phase-field variables $\phi$ and $\psi$ can be described by the conserved Cahn-Hilliard-type equations [14, 21],

$$
\begin{gathered}
\phi_{t}+\nabla \cdot(\mathbf{u} \phi)=\frac{1}{P e_{\phi}} \Delta w_{\phi}, \\
\psi_{t}+\nabla \cdot(\mathbf{u} \psi)=\frac{1}{P e_{\psi}} \nabla \cdot M_{\psi} \nabla w_{\psi},
\end{gathered}
$$

where $P e_{\phi}$ and $P e_{\psi}$ are Péclet numbers. A degenerate mobility $M_{\psi}=\psi(1-\psi)$, which vanishes at the extreme points $\psi=0$ and $\psi=1$, is adopted to combine with the logarithmic chemical potential $w_{\psi}$. Eqs. (2.6)-(2.9) are coupled to the Navier-Stokes equation in the form $[4,14]$

$$
\begin{gathered}
\rho \mathbf{u}_{t}+\rho \mathbf{u} \cdot \nabla \mathbf{u}+\mathbf{J} \cdot \nabla \mathbf{u}-\frac{1}{\operatorname{Re}} \nabla \cdot \eta D(\mathbf{u})+\nabla p+\frac{1}{\operatorname{ReCaCn}}\left(\phi \nabla w_{\phi}+\psi \nabla w_{\psi}\right)=0, \\
\nabla \cdot \mathbf{u}=0,
\end{gathered}
$$

where $D(\mathbf{u})=\nabla \mathbf{u}+\nabla^{T} \mathbf{u}$, and $\mathbf{J}=\left(\lambda_{\rho}-1\right) \nabla w_{\phi} / 2 P e_{\phi} . \mathbf{u}$ is the velocity field, $p$ is the pressure, $\mathrm{Re}$ is the Reynolds number and $\mathrm{Ca}$ is the Capillary number. We usually assume the density $\rho$ and viscosity $\eta$ has the following linear relations,

$$
\rho=\frac{1-\lambda_{\rho}}{2} \phi+\frac{1+\lambda_{\rho}}{2}, \quad \eta=\frac{1-\lambda_{\eta}}{2} \phi+\frac{1+\lambda_{\eta}}{2} .
$$

where $\lambda_{\rho}$ and $\lambda_{\eta}$ are density and viscosity ratios, respectively.

In particular, if we consider the body force, e.g., the gravitational force, the dimensionless momentum equation read

$$
\rho \mathbf{u}_{t}+\rho \mathbf{u} \cdot \nabla \mathbf{u}+\mathbf{J} \cdot \nabla \mathbf{u}-\frac{1}{\operatorname{Re}} \nabla \cdot \eta D(\mathbf{u})+\nabla p+\frac{1}{\mathrm{BoCn}}\left(\phi \nabla w_{\phi}+\psi \nabla w_{\psi}\right)-\rho \mathbf{g}=0
$$


where $\mathrm{Bo}=\mathrm{ReCa}$ is the Bond number, and $\mathrm{g}$ is the unit vector denoting the direction of body force.

Periodic boundary conditions or the following boundary conditions

$$
\partial_{n} \phi^{n+1}=\nabla w_{\phi}^{n+1} \cdot \mathbf{n}=\nabla w_{\psi}^{n+1} \cdot \mathbf{n}=\mathbf{u}=\partial_{n} p^{n+1}=0, \text { on } \Gamma,
$$

can be used to close the above governing system. Here $\Gamma$ denotes boundaries of the domain.

The total energy $E_{t o t}$ of the hydrodynamic system (2.5)-(2.10) is the sum of kinetic energy $E_{k}$ and free energy $E_{f}$

$$
\begin{aligned}
E_{\text {tot }}(\mathbf{u}, \phi, \psi, U, V)= & \int\left(\frac{\mathrm{We}}{2} \rho|\mathbf{u}|^{2}+\frac{\mathrm{Cn}^{2}}{4}|\nabla \phi|^{2}+\frac{\psi \phi^{2}}{4 \mathrm{Ex}}-\frac{\psi\left(\phi^{2}-1\right)^{2}}{4}\right) \mathrm{d} \Omega \\
& +U^{2}+\operatorname{Pi} V^{2}-\operatorname{Pi} B|\Omega|,
\end{aligned}
$$

where $\mathrm{We}=\mathrm{ReCaCn}$, and we can easily derive the following energy dissipation law.

$$
\begin{aligned}
\frac{d}{d t} E_{t o t}= & -\frac{1}{\mathrm{Pe}_{\phi}} \int\left|\nabla w_{\phi}\right|^{2} d \Omega \\
& -\frac{1}{\mathrm{Pe}_{\psi}} \int\left|\sqrt{M_{\psi}} \nabla w_{\psi}\right|^{2} d \Omega-\frac{\mathrm{CaCn}}{2} \int|\sqrt{\eta} D(\mathbf{u})|^{2} \mathrm{~d} \Omega \leq 0 .
\end{aligned}
$$

Next, we will develop an efficient time-marching scheme for the above governing system and carry out the energy estimate. To simplify the presentation, in the next section, we will take (2.9) as an example to construct the desired scheme.

\section{Numerical Scheme}

\subsection{Energy Stable First-Order Scheme}

We now present a first-order time-marching scheme to solve the governing system in Sect. 2. To deal with the case of nonmatching density, a cut-off function [4] is defined as

$$
\tilde{\phi}^{n+1}= \begin{cases}\phi^{n+1} & \left|\phi^{n+1}\right| \leq 1 \\ \operatorname{sign}\left(\phi^{n+1}\right) & \left|\phi^{n+1}\right|>1\end{cases}
$$

Given $\psi^{n}, \phi^{n}, \mathbf{u}^{n}$ and $p^{n}$, the scheme (3.1) calculates $\psi^{n+1}, \phi^{n+1}, \mathbf{u}^{n+1}$ and $p^{n+1}$ for $n \geq 0$ in three steps.

In step 1 , we update $\psi^{n+1}$ and $\phi^{n+1}$ by solving

$$
\frac{\psi^{n+1}-\psi^{n}}{\delta t}+\nabla \cdot\left(\mathbf{u}_{*}^{n} \psi^{n}\right)-\frac{1}{\mathrm{Pe}_{\psi}} \nabla \cdot M_{\psi}^{n} \nabla w_{\psi}^{n+1}=0,
$$




$$
\begin{gathered}
w_{\psi}^{n+1}=\frac{\operatorname{Pi} V^{n+1}}{\sqrt{E_{v}\left(\psi^{n}\right)}} G^{\prime}\left(\psi^{n}\right)+\frac{\left(\phi^{n}\right)^{2}}{4 \mathrm{Ex}}-\frac{\left(W^{n}\right)^{2}}{4}, \\
\frac{V^{n+1}-V^{n}}{\delta t}=\frac{1}{2 \sqrt{E_{v}\left(\psi^{n}\right)}} \int G^{\prime}\left(\psi^{n}\right) \frac{\psi^{n+1}-\psi^{n}}{\delta t} d \Omega, \\
\frac{\phi^{n+1}-\phi^{n}}{\delta t}+\nabla \cdot\left(\mathbf{u}_{*}^{n} \phi^{n}\right)-\frac{1}{\mathrm{Pe}_{\phi}} \Delta w_{\phi}^{n+1}=0, \\
w_{\phi}^{n+1}=-\frac{\mathrm{Cn}^{2}}{2} \Delta \phi^{n+1}+\frac{U^{n+1}}{\sqrt{E_{u}\left(\phi^{n}\right)}} F^{\prime}\left(\phi^{n}\right)+\frac{\psi^{n+1} \phi^{n+1}}{2 \mathrm{Ex}}-\frac{1}{2} \psi^{n+1} W^{n}\left(\phi^{n+1}+\phi^{n}\right), \\
\frac{U^{n+1}-U^{n}}{\delta t}=\frac{1 \mathrm{c})}{2 \sqrt{E_{u}\left(\phi^{n}\right)}} \int F^{\prime}\left(\phi^{n}\right) \frac{\phi^{n+1}-\phi^{n}}{\delta t} d \Omega,
\end{gathered}
$$

with periodic boundary conditions or the following boundary conditions

$$
\partial_{n} w_{\psi}^{n+1}=\partial_{n} \phi^{n+1}=\partial_{n} w_{\phi}^{n+1}=0, \text { on } \Gamma,
$$

where $\mathbf{u}_{*}^{n}$ is the intermediate velocity

$$
\mathbf{u}_{*}^{n}=\mathbf{u}^{n}-\frac{\delta t \psi^{n}}{\operatorname{We} \rho^{n}} \nabla w_{\psi}^{n+1}-\frac{\delta t \phi^{n}}{\operatorname{We} \rho^{n}} \nabla w_{\phi}^{n+1} .
$$

In step 2, we update $\mathbf{u}^{n+1}$ by solving [22]

$$
\left\{\begin{aligned}
\rho^{n} \frac{\mathbf{u}^{n+1}-\mathbf{u}^{n}}{\delta t}+ & \left(\rho^{n} \mathbf{u}_{*}^{n}\right) \cdot \nabla \mathbf{u}^{n+1}+\mathbf{J}^{n+1} \cdot \nabla \mathbf{u}^{n+1}-\frac{1}{\operatorname{Re}} \nabla \cdot \eta^{n} D\left(\mathbf{u}^{n+1}\right)+\nabla\left(2 p^{n}-p^{n-1}\right) \\
& +\frac{1}{\mathrm{We}}\left(\phi^{n} \nabla w_{\phi}^{n+1}+\psi^{n} \nabla w_{\psi}^{n+1}\right)+\frac{1+\lambda_{\rho}}{4}\left(\nabla \cdot \mathbf{u}_{*}^{n}\right) \mathbf{u}^{n+1}=0 \\
\mathbf{u}^{n+1}=0, \text { on } \Gamma &
\end{aligned}\right.
$$

where 


$$
\begin{gathered}
\mathbf{J}^{n+1}=\frac{\lambda_{\rho}-1}{2 \operatorname{Pe}_{\phi}} \nabla w_{\phi}^{n+1}, \quad \rho^{n+1}=\frac{1-\lambda_{\rho}}{2} \tilde{\phi}^{n+1}+\frac{1+\lambda_{\rho}}{2}, \\
\eta^{n+1}=\frac{1-\lambda_{\eta}}{2} \tilde{\phi}^{n+1}+\frac{1+\lambda_{\eta}}{2} .
\end{gathered}
$$

In step 3, we update $p^{n+1}$ by solving the pressure Poisson equation with a constant coefficient $[4,23]$

$$
\left\{\begin{array}{l}
-\Delta\left(p^{n+1}-p^{n}\right)=-\frac{\chi}{\delta t} \nabla \cdot \mathbf{u}^{n+1} \\
\nabla p^{n+1} \cdot \mathbf{n}=0, \text { on } \Gamma
\end{array}\right.
$$

where $\chi=\frac{1}{2} \min \left(1_{1}, \lambda_{\rho}\right)$.

Remark 3.1. (1) Computations of $\left(\psi^{n+1}, \phi^{n+1}\right), \mathbf{u}^{n+1}$ and $p^{n+1}$ are decoupled, which indicate that the scheme (3.1) is efficient and easy-to-implement. At each time step, $u^{n+1}$ and $p^{n+1}$ can be obtained by solving only two elliptic equations; Moreover, $V^{n+1}$ and $U^{n+1}$ do not involve any extra computational cost, since they can be calculated explicitly once we obtain $\psi^{n+1}$ and $\phi^{n+1}$. (2) In the explicit convective velocity $\mathbf{u}_{*}^{n}$, we introduce a firstorder stabilization term [24], which plays a dominant role in decoupling the computation of $\left(\psi^{n+1}, \phi^{n+1}\right)$ from $u^{n+1}$ and constructing the unconditionally energy stable scheme.

Theorem 3.1. The scheme (3.1) is unconditionally energy stable, and satisfies the following discrete energy dissipation law:

$$
\begin{aligned}
& E_{\text {tot }}^{n+1}-E_{\text {tot }}^{n} \leq \\
& \quad-\frac{\delta t}{P e_{\psi}}\left\|\sqrt{M_{\psi}^{n}} \nabla w_{\psi}^{n+1}\right\|^{2}-\frac{\delta t}{P e_{\phi}}\left\|\nabla w_{\phi}^{n+1}\right\|^{2}-\frac{\delta t \mathrm{CaCn}}{2}\left\|\sqrt{\eta^{n}} D\left(\mathbf{u}^{n+1}\right)\right\|^{2} \leq 0,
\end{aligned}
$$

where

$$
\begin{aligned}
E_{t o t}^{n}= & \frac{\mathrm{We}}{2}\left(\rho^{n},\left|\mathbf{u}^{n}\right|^{2}\right)+\frac{\delta t^{2} \mathrm{We}}{2 \chi}\left\|\nabla p^{n}\right\|^{2}+\frac{\mathrm{Cn}^{2}}{4}\left\|\nabla \phi^{n}\right\|^{2}+\left(U^{n}\right)^{2}+\operatorname{Pi}\left(V^{n}\right)^{2} \\
& +\frac{1}{4 \mathrm{Ex}}\left(\psi^{n},\left|\phi^{n}\right|^{2}\right)-\frac{1}{4}\left(\psi^{n},\left|W^{n}\right|^{2}\right)-\operatorname{Pi} B|\Omega|,
\end{aligned}
$$

here $\|\cdot\|$ denotes the $\mathrm{L}^{2}$-norm in $\Omega$. Now we will rigorously prove the discrete energy dissipation law in (3.2). We first introduce an intermediate kinetic energy [25] as

$$
E_{k, *}^{n}=\frac{\mathrm{We}}{2}\left(\rho^{n} \mathbf{u}_{*}^{n}, \mathbf{u}_{*}^{n}\right)
$$

The difference between $E_{k}^{n+1}$ and $E_{k, *}^{n}$ is estimated as 


$$
\begin{aligned}
E_{k}^{n+1} & -E_{k, *}^{n}=\frac{\mathrm{We}}{2}\left(\rho^{n+1},\left|\mathbf{u}^{n+1}\right|^{2}\right)-\frac{\mathrm{We}}{2}\left(\rho^{n},\left|\mathbf{u}_{*}^{n}\right|^{2}\right) \\
& =\frac{\mathrm{We}}{2}\left(\rho^{n},\left|\mathbf{u}^{n+1}\right|^{2}-\left|\mathbf{u}_{*}^{n}\right|^{2}\right)+\frac{\mathrm{We}}{2}\left(\rho^{n+1}-\rho^{n},\left|\mathbf{u}^{n+1}\right|^{2}\right) \\
& =\mathrm{We}\left(\rho^{n}\left(\mathbf{u}^{n+1}-\mathbf{u}_{*}^{n}\right), \mathbf{u}^{n+1}\right)-\frac{\mathrm{We}}{2}\left(\rho^{n},\left|\mathbf{u}^{n+1}-\mathbf{u}_{*}^{n}\right|^{2}\right)+\frac{\mathrm{We}}{2}\left(\rho^{n+1}-\rho^{n},\left|\mathbf{u}^{n+1}\right|^{2}\right) .
\end{aligned}
$$

Substituting (3.1i) into (3.1a), we obtain the following identity

$$
\rho^{n+1}-\rho^{n}=-\frac{\delta t\left(1-\lambda_{\rho}\right)}{2} \nabla \cdot\left(\phi^{n} \mathbf{u}_{*}^{n}\right)-\delta t \nabla \cdot \mathbf{J}^{n+1} .
$$

We can also easily derive from $(3.1 \mathrm{~g})$ that

$$
\mathrm{We} \rho^{n}\left(\mathbf{u}^{n+1}-\mathbf{u}^{n}\right)+\delta t\left(\psi^{n} \nabla w_{\psi}^{n+1}+\phi \nabla w_{\phi}^{n+1}\right)=\mathrm{W} \mathrm{e} \rho^{n}\left(\mathbf{u}^{n+1}-\mathbf{u}_{*}^{n}\right) .
$$

Using the identity (3.6), we have

$$
\begin{array}{r}
\mathrm{We} \rho^{n}\left(\mathbf{u}^{n+1}-\mathbf{u}_{*}^{n}\right)=\delta t \operatorname{CaCn} \nabla \cdot \eta^{n} D\left(\mathbf{u}^{n+1}\right)-\delta t \mathrm{We} \nabla\left(2 p^{n}-p^{n-1}\right)-\delta t \mathrm{We}\left(\rho^{n} \mathbf{u}_{*}^{n}\right) \cdot \nabla \mathbf{u}^{n+1} \\
-\delta t \mathrm{We} \mathbf{J}^{n+1} \cdot \nabla \mathbf{u}^{n+1}-\frac{\delta t \mathrm{We}\left(1+\lambda_{\rho}\right)}{4}\left(\nabla \cdot \mathbf{u}_{*}^{n}\right) \mathbf{u}^{n+1}
\end{array}
$$

By taking the $L^{2}$ inner product of (3.7) with $u^{n+1}$, and using (3.4) and the following identities

$$
\begin{gathered}
-\delta t \mathrm{We}\left(\left(\rho^{n} \mathbf{u}_{*}^{n}\right) \cdot \nabla \mathbf{u}^{n+1}, \mathbf{u}^{n+1}\right)=\frac{\delta t \mathrm{We}}{2}\left(\nabla \cdot\left(\rho^{n} \mathbf{u}_{*}^{n}\right),\left|\mathbf{u}^{n+1}\right|^{2}\right) \\
=\frac{\delta t \mathrm{We}\left(1-\lambda_{\rho}\right)}{4}\left(\nabla \cdot\left(\phi^{n} \mathbf{u}_{*}^{n}\right),\left|\mathbf{u}^{n+1}\right|^{2}\right)+\frac{\delta t \mathrm{We}\left(1+\lambda_{\rho}\right)}{4}\left(\nabla \cdot \mathbf{u}_{*}^{n},\left|\mathbf{u}^{n+1}\right|^{2}\right) \\
\left(\left(\mathbf{J}^{n+1} \cdot \nabla\right) \mathbf{u}^{n+1}+\frac{1}{2}\left(\nabla \cdot \mathbf{J}^{n+1}\right) \mathbf{u}^{n+1}, \mathbf{u}^{n+1}\right)=0
\end{gathered}
$$


we can derive that

$$
\begin{aligned}
E_{k}^{n+1} & -E_{k, *}^{n}=-\frac{\delta t \mathrm{CaCn}}{2}\left\|\sqrt{\eta^{n}} D\left(\mathbf{u}^{n+1}\right)\right\|^{2}-\delta t \mathrm{We}\left(\left(\rho^{n} \mathbf{u}_{*}^{n}\right) \cdot \nabla \mathbf{u}^{n+1}, \mathbf{u}^{n+1}\right) \\
& -\delta t \mathrm{We}\left(\mathbf{J}^{n+1} \cdot \nabla \mathbf{u}^{n+1}, \mathbf{u}^{n+1}\right)-\frac{\delta t \mathrm{We}(1+\lambda \rho)}{4}\left(\nabla \cdot \mathbf{u}_{*}^{n},\left|\mathbf{u}^{n+1}\right|^{2}\right) \\
& -\delta t \mathrm{We}\left(p^{n+1}-2 p^{n}+p^{n-1}, \nabla \cdot \mathbf{u}^{n+1}\right)+\delta t \mathrm{We}\left(p^{n+1}, \nabla \cdot \mathbf{u}^{n+1}\right) \\
& -\frac{\mathrm{We}}{2}\left(\rho^{n},\left|\mathbf{u}^{n+1}-\mathbf{u}_{*}^{n}\right|^{2}\right)-\frac{\delta t \mathrm{We}}{2}\left(\frac{1-\lambda \rho}{2} \nabla \cdot\left(\phi^{n} \mathbf{u}_{*}^{n}\right)+\nabla \cdot \mathbf{J}^{n+1},\left|\mathbf{u}^{n+1}\right|^{2}\right) \\
& =-\frac{\delta t \mathrm{CaCn}}{2}\left\|\sqrt{\eta^{n}} D\left(\mathbf{u}^{n+1}\right)\right\|^{2}-\delta t \mathrm{We}\left(p^{n+1}-2 p^{n}+p^{n-1}, \nabla \cdot \mathbf{u}^{n+1}\right) \\
& +\delta t \mathrm{We}\left(p^{n+1}, \nabla \cdot \mathbf{u}^{n+1}\right)-\frac{\mathrm{We}}{2}\left(\rho^{n},\left|\mathbf{u}^{n+1}-\mathbf{u}_{*}^{n}\right|^{2}\right) .
\end{aligned}
$$

Using the Eq. (3.1g), we obtain

$$
\begin{aligned}
E_{k, *}^{n} & -E_{k}^{n}=\frac{\mathrm{We}}{2}\left(\rho^{n},\left|\mathbf{u}_{*}^{n}\right|^{2}-\left|\mathbf{u}^{n}\right|^{2}\right)=\left(\mathrm{We} \rho^{n}\left(\mathbf{u}_{*}^{n}-\mathbf{u}^{n}\right), \mathbf{u}_{*}^{n}\right)-\frac{\mathrm{We}}{2}\left(\rho^{n},\left|\mathbf{u}_{*}^{n}-\mathbf{u}^{n}\right|^{2}\right) \\
& =-\delta t\left(\psi^{n} \nabla \cdot w_{\psi}^{n+1}+\phi^{n} \nabla w_{\phi}^{n+1}, \mathbf{u}_{*}^{n}\right)-\frac{\mathrm{We}}{2}\left(\rho^{n},\left|\mathbf{u}_{*}^{n}-\mathbf{u}^{n}\right|^{2}\right) \\
& =\delta t\left(\nabla \cdot\left(\psi \mathbf{u}_{*}^{n}\right), w_{\psi}^{n+1}\right)+\delta t\left(\nabla \cdot\left(\phi \mathbf{u}_{*}^{n}\right), w_{\phi}^{n+1}\right)-\frac{\mathrm{We}}{2}\left(\rho^{n},\left|\mathbf{u}_{*}^{n}-\mathbf{u}^{n}\right|^{2}\right) .
\end{aligned}
$$

Summing up Eqs. (3.8) and (3.9), we get

$$
\begin{aligned}
E_{k}^{n+1} & -E_{k}^{n}=-\frac{\delta t \mathrm{CaCn}}{2}\left\|\sqrt{\eta^{n}} D\left(\mathbf{u}^{n+1}\right)\right\|^{2}-\delta t \mathrm{We}\left(p^{n+1}-2 p^{n}+p^{n-1}, \nabla \cdot \mathbf{u}^{n+1}\right) \\
& +\delta t \mathrm{We}\left(p^{n+1}, \nabla \cdot \mathbf{u}^{n+1}\right)+\delta t\left(\nabla \cdot\left(\psi \mathbf{u}_{*}^{n}\right), w_{\psi}^{n+1}\right)+\delta t\left(\nabla \cdot\left(\phi \mathbf{u}_{*}^{n}\right), w_{\phi}^{n+1}\right) \\
& -\frac{\mathrm{We}}{2}\left(\rho^{n},\left|\mathbf{u}^{n+1}-\mathbf{u}_{*}^{n}\right|^{2}\right)-\frac{\mathrm{We}}{2}\left(\rho^{n},\left|\mathbf{u}_{*}^{n}-\mathbf{u}^{n}\right|^{2}\right) .
\end{aligned}
$$

By taking the $L^{2}$ inner product of (3.1j) with $\delta t^{2} \mathrm{We}\left(p^{n+1}-2 p^{n}+p^{n}-1\right) / \chi$ and with$\delta t^{2} \mathrm{We} p^{n+1} / \chi$ separately, we obtain

$$
\begin{array}{r}
-\frac{\delta t^{2} \mathrm{We}}{2 \chi}\left(\left\|\nabla\left(p^{n+1}-p^{n}\right)\right\|^{2}-\left\|\nabla\left(p^{n}-p^{n-1}\right)\right\|^{2}+\left\|\nabla\left(p^{n+1}-2 p^{n}+p^{n-1}\right)\right\|^{2}\right) \\
=\delta t \mathrm{We}\left(p^{n+1}-2 p^{n}+p^{n-1}, \nabla \cdot \mathbf{u}^{n+1}\right),
\end{array}
$$


and

$$
\frac{\delta t^{2} \mathrm{We}}{2 \chi}\left(\left\|\nabla p^{n+1}\right\|^{2}-\left\|\nabla p^{n}\right\|^{2}+\left\|\nabla\left(p^{n+1}-p^{n}\right)\right\|^{2}\right)=-\delta t \mathrm{We}\left(p^{n+1}, \nabla \cdot \mathbf{u}^{n+1}\right) .
$$

Combining (3.11) and (3.12), yields

$$
\begin{aligned}
& -\delta t \mathrm{We}\left(p^{n+1}-2 p^{n}+p^{n-1}, \nabla \cdot \mathbf{u}^{n+1}\right)+\delta t \mathrm{We}\left(p^{n+1}, \nabla \cdot \mathbf{u}^{n+1}\right) \\
& =-\frac{\delta t^{2} \mathrm{We}}{2 \chi}\left(\left\|\nabla p^{n+1}\right\|^{2}-\left\|\nabla p^{n}\right\|^{2}\right)-\frac{\delta t^{2} \mathrm{We}}{2 \chi}\left\|\nabla\left(p^{n}-p^{n-1}\right)\right\|^{2} \\
& +\frac{\delta t^{2} \mathrm{We}}{2 \chi}\left\|\nabla\left(p^{n+1}-2 p^{n}+p^{n-1}\right)\right\|^{2} .
\end{aligned}
$$

We take the difference of (3.1j) at step $t^{n+1}$ and $t^{n}$, pair the resulting equation with $\delta t^{2} \mathrm{We}\left(p^{n+1}-2 p^{n}+p^{n}-1\right) /(2 \chi)$ then take integration by parts for both sides to derive

$$
\frac{\delta t^{2} \mathrm{We}}{2 \chi}\left\|\nabla\left(p^{n+1}-2 p^{n}+p^{n-1}\right)\right\|^{2} \leq \frac{\chi}{2} \mathrm{We}\left\|\mathbf{u}^{n+1}-\mathbf{u}^{n}\right\|^{2} \leq \frac{\mathrm{We}}{4}\left(\rho^{n},\left|\mathbf{u}^{n+1}-\mathbf{u}^{n}\right|\right)^{2}
$$

Summing up Eqs. (3.10), (3.13) and (3.14), and using the triangle inequality

$$
\frac{\mathrm{We}}{2}\left(\rho^{n},\left|\mathbf{u}^{n+1}-\mathbf{u}_{*}^{n}\right|^{2}\right)+\frac{\mathrm{We}}{2}\left(\rho^{n},\left|\mathbf{u}_{*}^{n}-\mathbf{u}^{n}\right|^{2}\right) \geq \frac{\mathrm{W} \mathrm{e}}{4}\left(\rho^{n},\left|\mathbf{u}^{n+1}-\mathbf{u}^{n}\right|\right)^{2} .
$$

we can derive that

$$
\begin{aligned}
E_{k}^{n+1}-E_{k}^{n} & \leq-\frac{\delta t \mathrm{CaCn}}{2}\left\|\sqrt{\eta^{n}} D\left(\mathbf{u}^{n+1}\right)\right\|^{2}-\frac{\delta t^{2} \mathrm{We}}{2 \chi}\left(\left\|\nabla p^{n+1}\right\|^{2}-\left\|\nabla p^{n}\right\|^{2}\right) \\
& +\delta t\left(\nabla \cdot\left(\psi \mathbf{u}_{*}^{n}\right), w_{\psi}^{n+1}\right)+\delta t\left(\nabla \cdot\left(\phi \mathbf{u}_{*}^{n}\right), w_{\phi}^{n+1}\right) .
\end{aligned}
$$

By taking the inner product of (3.1a) with $\delta t w_{\psi}^{n+1}$, we can easily derive that

$$
\left(\psi^{n+1}-\psi^{n}, w_{\psi}^{n+1}\right)+\delta t\left(\nabla \cdot\left(\mathbf{u}_{*}^{n} \psi^{n}\right), w_{\psi}^{n+1}\right)=-\frac{\delta t}{P e_{\psi}}\left\|\sqrt{M_{\psi}^{n}} \nabla w_{\psi}^{n+1}\right\|^{2}
$$

By taking the inner product of (3.1b) with $-\left(\psi^{n+1}-\psi^{n}\right)$, we can derive that

$$
\begin{aligned}
-\left(\psi^{n+1}-\psi^{n}, w_{\psi}^{n+1}\right)= & -\operatorname{Pi}\left(V^{n+1} a^{n}, \psi^{n+1}-\psi^{n}\right)-\frac{1}{4 \mathrm{Ex}}\left(\left|\phi^{n}\right|^{2}, \psi^{n+1}-\psi^{n}\right) \\
& +\frac{1}{4}\left(\left|W^{n}\right|^{2}, \psi^{n+1}-\psi^{n}\right) .
\end{aligned}
$$


where $a^{n}=G^{\prime}\left(\psi^{n}\right) / \sqrt{E_{v}\left(\psi^{n}\right)}$. Taking the inner product of (3.1c) with $2 \delta t \mathrm{Pi} V^{n+1}$ to obtain

$$
\operatorname{Pi}\left[\left(V^{n+1}\right)^{2}-\left(V^{n}\right)^{2}+\left(V^{n+1}-V^{n}\right)^{2}\right]=\operatorname{Pi}\left(V^{n+1} a^{n}, \psi^{n+1}-\psi^{n}\right) .
$$

Summing up Eqs. (3.17)-(3.19), we get

$$
\begin{aligned}
& \operatorname{Pi}\left[\left(V^{n+1}\right)^{2}-\left(V^{n}\right)^{2}+\left(V^{n+1}-V^{n}\right)^{2}\right]=-\delta t\left(\nabla\left(\mathbf{u}_{*}^{n} \psi^{n}\right), w_{\psi}^{n+1}\right)-\frac{\delta t}{P e_{\psi}}\left\|\sqrt{M_{\psi}^{n}} \nabla w_{\psi}^{n+1}\right\|^{2} \\
& -\frac{1}{4 \mathrm{Ex}}\left(\left|\phi^{n}\right|^{2}, \psi^{n+1}-\psi^{n}\right)+\frac{1}{4}\left(\left|W^{n}\right|^{2}, \psi^{n+1}-\psi^{n}\right) .
\end{aligned}
$$

By taking the inner product of (3.1d) with $\delta t w_{\phi}^{n+1}$, we have

$$
\left(\phi^{n+1}-\phi^{n}, w_{\phi}^{n+1}\right)+\delta t\left(\nabla \cdot\left(\mathbf{u}_{*}^{n} \phi^{n}\right), w_{\phi}^{n+1}\right)=-\frac{\delta t}{P e_{\phi}}\left\|\nabla w_{\phi}^{n+1}\right\|^{2} .
$$

By taking the inner product of (3.1e) with $-\left(\phi^{n+1}-\phi^{n}\right)$, we can derive that

$$
\begin{aligned}
& -\left(\phi^{n+1}-\phi^{n}, w_{\phi}^{n+1}\right)=-\frac{\mathrm{Cn}^{2}}{2}\left(\nabla \phi^{n+1}, \nabla \phi^{n+1}-\nabla \phi^{n}\right)-\left(U^{n+1} b^{n}, \phi^{n+1}-\phi^{n}\right) \\
& -\frac{1}{2 \mathrm{Ex}}\left(\psi^{n+1} \phi^{n+1}, \phi^{n+1}-\phi^{n}\right)+\frac{1}{2}\left(\psi^{n+1} W^{n}\left(\phi^{n+1}+\phi^{n}\right), \phi^{n+1}-\phi^{n}\right) \\
& =-\frac{\mathrm{Cn}^{2}}{4}\left(\left\|\nabla \phi^{n+1}\right\|^{2}-\left\|\nabla \phi^{n}\right\|^{2}+\left\|\nabla \phi^{n+1}-\nabla \phi^{n}\right\|^{2}\right)-\left(U^{n+1} b^{n}, \phi^{n+1}-\phi^{n}\right) \\
& -\frac{1}{4 \mathrm{Ex}}\left[\left(\psi^{n+1},\left|\phi^{n+1}\right|^{2}\right)-\left(\psi^{n+1},\left|\phi^{n}\right|^{2}\right)+\left(\psi^{n+1},\left|\phi^{n+1}-\phi^{n}\right|^{2}\right)\right] \\
& +\frac{1}{4}\left[\left(\psi^{n+1},\left|W^{n+1}\right|^{2}\right)-\left(\psi^{n+1},\left|W^{n}\right|^{2}\right)-\left(\psi^{n+1},\left|W^{n+1}-W^{n}\right|^{2}\right)\right] .
\end{aligned}
$$

where $b^{n}=F^{\prime}\left(\phi^{n}\right) / \sqrt{E_{u}\left(\phi^{n}\right)}$. Taking the inner product of (3.1f) with $2 \delta t U^{n+1}$ to obtain

$$
\left[\left(U^{n+1}\right)^{2}-\left(U^{n}\right)^{2}+\left(U^{n+1}-U^{n}\right)^{2}\right]=\left(U^{n+1} b^{n}, \phi^{n+1}-\phi^{n}\right) .
$$

Summing up Eqs. (3.20)-(3.23), and dropping off some positive terms, we have 


$$
\begin{aligned}
& \frac{\mathrm{Cn}^{2}}{4}\left(\left\|\nabla \phi^{n+1}\right\|^{2}-\left\|\nabla \phi^{n}\right\|^{2}\right)+\left[\left(U^{n+1}\right)^{2}-\left(U^{n}\right)^{2}\right]+\operatorname{Pi}\left[\left(V^{n+1}\right)^{2}-\left(V^{n}\right)^{2}\right] \\
& +\frac{1}{4 \mathrm{Ex}}\left[\left(\psi^{n+1},\left|\phi^{n+1}\right|^{2}\right)-\left(\psi^{n},\left|\phi^{n}\right|^{2}\right)\right]-\frac{1}{4}\left[\left(\psi^{n+1},\left|W^{n+1}\right|^{2}\right)-\left(\psi^{n},\left|W^{n}\right|^{2}\right)\right] \\
& \leq-\frac{\delta t}{P e_{\psi}}\left\|\sqrt{M_{\psi}^{n}} \nabla w_{\psi}^{n+1}\right\|^{2}-\frac{\delta t}{P e_{\psi}}\left\|\nabla w_{\psi}^{n+1}\right\|^{2}-\delta t\left(\nabla\left(\mathbf{u}_{*}^{n} \psi^{n}\right), w_{\psi}^{n+1}\right)-\delta t\left(\nabla\left(\mathbf{u}_{*}^{n} \phi^{n}\right), w_{\phi}^{n+1}\right) .
\end{aligned}
$$

Finally, combining (3.16) and (3.24), we arrive at the desired result.

\section{Numerical Results}

To implement the scheme (3.1), we use a finite difference method on staggered grids to discretize space. We pay special attention to the discretization of the convection terms in the Cahn-Hilliard and Navier-Stokes equations. A composite high resolution scheme, known as the MINMOD scheme, is used to reduce the undershoot and overshoot around the interface. The computations of $\psi^{n+1}, \phi^{n+1}, \mathbf{u}^{n+1}$ and $p^{n+1}$ can be totally decoupled if we replace $\psi^{n+1}$ in (3.1e) with $\psi^{n}$. The simplified scheme is extremely efficient and easy-to-implement. However, this simplification will definitely destroy the unconditional energy stability of our scheme. The implementation of such a simplified scheme requires small time step-sizes to obtain the desired accuracy and energy stability. The above scheme is adopted in [26] and numerical results demonstrate the energy stability of the proposed scheme. Here we will not present these results due to the limit of article length.

We simulate the droplet deformation under the horizontal body force and a shear flow in a computational domain $\Omega=[0,3] \times[0,1]$. Periodic boundary conditions are applied on the left and right sides. A circular droplet with the radius of $r=0.3$ is initially placed at $(1,0.5)$. Other simulation parameters are listed as follows:

$$
\begin{aligned}
P e_{\phi} & =10, P e_{\psi}=100, \operatorname{Re}=10, B o=1, \mathrm{Cn}=0.01, \mathrm{Ex}=1, \mathrm{Pi}=0.1227, \lambda_{\rho} \\
& =\lambda_{v}=10 .
\end{aligned}
$$

Figure 1 shows the time evolution plots of droplet deformation and surfactant concentration. The droplet continuously deforms and moves forward under the action of the shear flow and the body force. We can divide the whole process into two stages based on the droplet deformation and surfactant migration. At the first stage, the body force has limited effect on the droplet deformation compared with the shear flow. Surfactants gradually migrate toward droplet tips, as shown in Fig. 1(b), resulting in the non-uniformity of interfacial tension along the interface. As we mentioned before, the surfactant concentration gradient induces the Marangoni stress, which will resist the further migration of surfactants. However, the Marangoni stress is not large enough to balance the effect of shear flow, and surfactants continue to move toward tips. In Fig. 1 (c), surfactants are swept into the bulk phases when concentration reaches the maximum at the droplet tips. At the second stage, the body force plays an important role in 
the droplet deformation and surfactant migration. In Fig. 1(d), surfactants on the tip A are slowly swept towards the ABC segment under the effect of the body force. Surfactants along the ADC segment continuously move to the tips under the combined action of the shear flow and the body force.

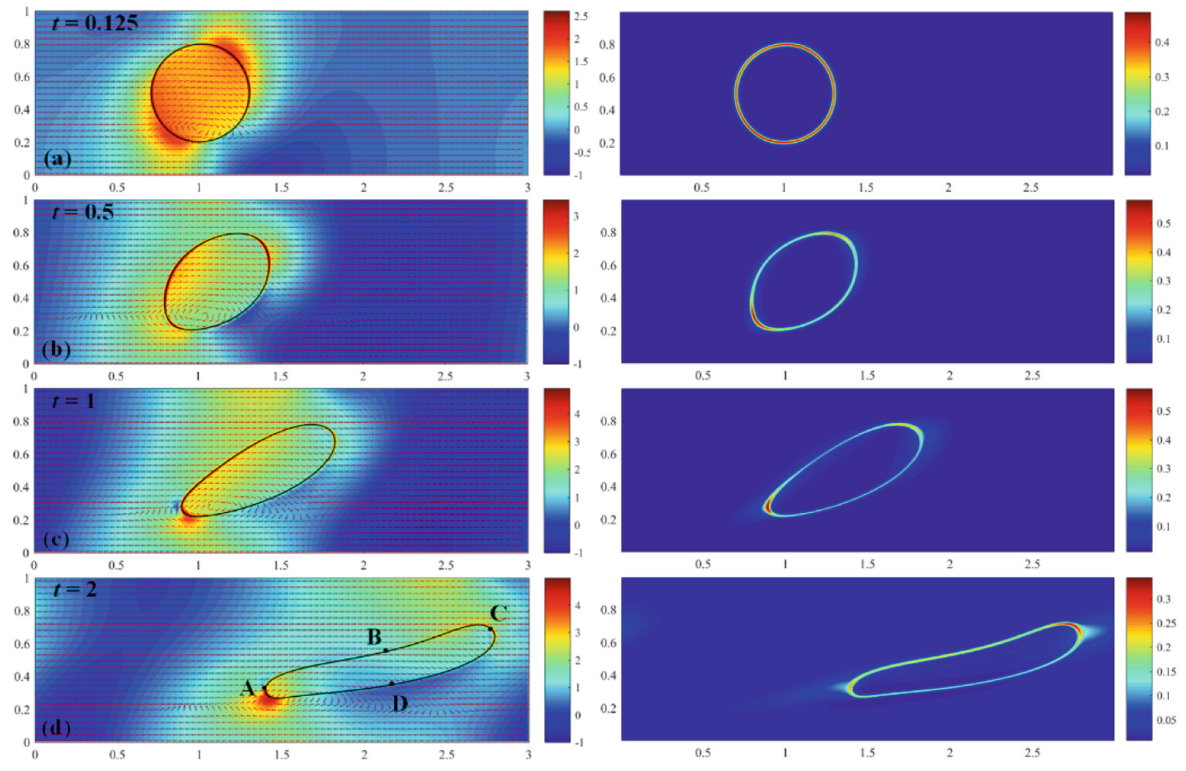

Fig. 1. Evolutions of pressure field (background color), quiver plot of velocity $(u, v)$, phase-field variables $\phi$ and $\psi$. For each subfigure, the right is the profile of $\psi .\left(\psi_{b}=1.5 \times 10^{-2}\right)$.

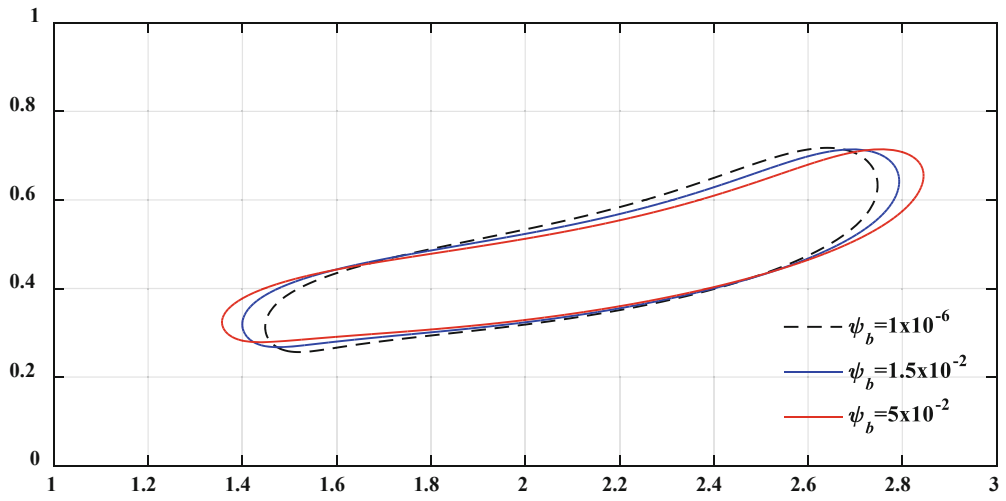

Fig. 2. Profiles of phase-field variable $\phi$ at $t=1$ (left) and $t=2$ (right). (black dash line: $\psi_{b}=1 \times 10^{-6}$; blue solid line: $\psi_{b}=1.5 \times 10^{-2}$; red solid line: $\psi_{b}=5 \times 10^{-2}$ ) (Color figure online) 
Figure 2 demonstrates the profiles of phase-field variable $\phi$ at three different $\psi_{b}$ values. A more prolate profile of $\phi$ is observed for a higher surfactant bulk concentration, which confirms the effect of surfactants in reducing the interfacial tension.

\section{Conclusion}

The numerical approximation of incompressible and immiscible two-phase flows with soluble surfactants is the main topic in this paper. An efficient, accurate and energy stable time-marching scheme is constructed using the SAV approach for the hydrodynamics coupled phase-field surfactant model with variable densities. We rigorously prove the unconditional energy stability of the semi-implicit scheme. Numerical results demonstrate the energy stability of the proposed scheme.

\section{References}

1. Khatri, S., Tornberg, A.-K.: An embedded boundary method for soluble surfactants with interface tracking for two-phase flows. J. Comput. Phys. 256, 768-790 (2014)

2. Yang, X.: Numerical approximations for the Cahn-Hilliard phase field model of the binary fluid-surfactant system. J. Sci. Comput. 74, 1533-1553 (2018)

3. Liu, H., Zhang, Y.: Phase-field modeling droplet dynamics with soluble surfactants. J. Comput. Phys. 229(24), 9166-9187 (2010)

4. Shen, J., Yang, X.: Decoupled, energy stable schemes for phase-field models of two-phase incompressible flows. SIAM J. Numer. Anal. 53(1), 279-296 (2015)

5. Yue, P., Feng, J.J., Liu, C., Shen, J.: A diffuse-interface method for simulating two-phase flows of complex fluids. J. Fluid Mech. 515, 293-317 (2014)

6. Zhu, G., Kou, J., Yao, J., Li, A., Sun, S.: A phase-field moving contact line model with soluble surfactants. J. Comput. Phys. 405, 109170 (2020)

7. Xu, X., Di, Y., Yu, H.: Sharp-interface limits of a phase-field model with a generalized Navier slip boundary condition for moving contact lines. J. Fluid Mech. 849, 805-833 (2018)

8. Zhu, G., Chen, H., Yao, J., Sun, S.: Efficient energy-stable schemes for the hydrodynamics coupled phase-field model. Appl. Math. Model. 70, 82-108 (2019)

9. Laradji, M., Guo, H., Grant, M., Zuckermann, M.J.: The effect of surfactants on the dynamics of phase separation. J. Phys.: Condens. Matt. 4(32), 6715 (1992)

10. Li, Y., Kim, J.: A comparison study of phase-field models for an immiscible binary mixture with surfactant. Eur. Phys. J. B 85(10), 340 (2012)

11. Yang, X., Ju, L.: Linear and unconditionally energy stable schemes for the binary fluidsurfactant phase field model. Comput. Method Appl. M 318, 1005-1029 (2017)

12. Zhu, G., Kou, J., Sun, S., Yao, J., Li, A.: Decoupled, energy stable schemes for a phase-field surfactant model. Comput. Phys. Commun. 233, 67-77 (2018)

13. Van der Sman, R., Van der Graaf, S.: Diffuse interface model of surfactant adsorption onto flat and droplet interfaces. Rheol. Acta 46(1), 3-11 (2016)

14. Engblom, S., Do-Quang, M., Amberg, G., Tornberg, A.-K.: On diffuse interface modeling and simulation of surfactants in two-phase fluid flow. Commun. Comput. Phys. 14(4), 879915 (2013) 
15. Booty, M., Siegel, M.: A hybrid numerical method for interfacial fluid flow with soluble surfactant. J. Comput. Phys. 229(10), 3864-3883 (2010)

16. Pätzold, G., Dawson, K.: Numerical simulation of phase separation in the presence of surfactants and hydrodynamics. Phys. Rev. E 52(6), 6908 (1995)

17. Teigen, K.E., Song, P., Lowengrub, J., Voigt, A.: A diffuse-interface method for two-phase flows with soluble surfactants. J. Comput. Phys. 230(2), 375-393 (2011)

18. Yun, A., Li, Y., Kim, J.: A new phase-field model for a water-oil-surfactant system. Appl. Math. Comput. 229, 422-432 (2014)

19. Zhu, G., Kou, J., Sun, S., Yao, J., Li, A.: Numerical approximation of a phase-field surfactant model with fluid flow. J. Sci. Comput. 80(1), 223-247 (2019)

20. Shen, J., Xu, J., Yang, J.: The scalar auxiliary variable (SAV) approach for gradient flows. J. Comput. Phys. 353, 407-416 (2018)

21. Zhu, G., Kou, J., Yao, B., Wu, Y.-S., Yao, J., Sun, S.: Thermodynamically consistent modelling of two-phase flows with moving contact line and soluble surfactants. J. Fluid Mech. 879, 327-359 (2019)

22. Feng, X., Kou, J., Sun, S.: A novel energy stable numerical scheme for Navier-Stokes-CahnHilliard two-phase flow model with variable densities and viscosities. In: Shi, Y., et al. (eds.) ICCS 2018. LNCS, vol. 10862, pp. 113-128. Springer, Cham (2018). https://doi.org/10. 1007/978-3-319-93713-7_9

23. Gao, M., Wang, X.-P.: An efficient scheme for a phase field model for the moving contact line problem with variable density and viscosity. J. Comput. Phys. 272, 704-718 (2014)

24. Shen, J., Yang, X.: Decoupled energy stable schemes for phase-field models of two-phase complex fluids. SIAM J. Sci. Comput. 36(1), B122-B145 (2014)

25. Kou, J., Sun, S.: Thermodynamically consistent modeling and simulation of multicomponent two-phase flow with partial miscibility. Comput. Method Appl. M 331, 623-649 (2018)

26. Zhu, G., Li, A.: Interfacial dynamics with soluble surfactants: a phase-field two-phase flow model with variable densities. Adv. Geo-Energy Res. 4(1), 86-98 (2020) 